\title{
Adjoint master equation for quantum Brownian motion
}

\author{
Matteo Carlesso* and Angelo Bassi ${ }^{\dagger}$ \\ Department of Physics, University of Trieste, Strada Costiera 11, 34151 Trieste, Italy \\ and Istituto Nazionale di Fisica Nucleare, Trieste Section, Via Valerio 2, 34127 Trieste, Italy
}

(Received 28 January 2017; published 23 May 2017)

\begin{abstract}
Quantum Brownian motion is a fundamental model for a proper understanding of open quantum systems in different contexts such as chemistry, condensed-matter physics, biophysics, and optomechanics. In this paper we propose a different approach to describe this model. We provide an exact and analytic equation for the time evolution of the operators and we show that the corresponding equation for the states is equivalent to well-known results in the literature. The dynamics is expressed in terms of the spectral density, regardless of the strength of the coupling between the system and the bath. Our derivation allows to compute the time evolution of physically relevant quantities in a much easier way than previous formulations. An example is explicitly studied.
\end{abstract}

DOI: 10.1103/PhysRevA.95.052119

\section{INTRODUCTION}

Technical improvements in quantum experiments are making impressive steps forward, reaching levels of accuracy that were hardly imaginable a few decades ago. Controlling the noise is often the crucial challenge for further progress, and a theoretical understanding is important to disentangle environmental effects from intrinsic properties of the system.

Quantum Brownian motion [1-6] is the paradigm of an open quantum system interacting with an external bath, and nowadays it finds applications in several physical contexts such as chemistry [7], condensed matter [8-10], biophysics [11-14], and optomechanics [15-18], to name a few.

The model consists of a particle $S$ of mass $M$, with position $\hat{x}$ and momentum $\hat{p}$, harmonically trapped at frequency $\omega_{\mathrm{S}}$ and interacting with a thermal bath of independent harmonic oscillators, with positions $\hat{R}_{k}$, momenta $\hat{P}_{k}$, mass $m$, and frequencies $\omega_{k}$. This model has become a milestone in the theory of open quantum system [2,19-34]. The total Hamiltonian $\hat{H}_{\mathrm{T}}$ of system plus bath is $\hat{H}_{\mathrm{T}}=\hat{H}_{\mathrm{S}}+\hat{H}_{\mathrm{I}}+\hat{H}_{\mathrm{B}}$, where

$$
\begin{aligned}
& \hat{H}_{\mathrm{S}}=\frac{\hat{p}^{2}}{2 M}+\frac{1}{2} M \omega_{\mathrm{S}}^{2} \hat{x}^{2}, \\
& \hat{H}_{\mathrm{B}}=\sum_{k} \frac{\hat{P}_{k}^{2}}{2 m}+\frac{1}{2} m \omega_{k}^{2} \hat{R}_{k}^{2}, \\
& \hat{H}_{\mathrm{I}}=\hat{x} \sum_{k} C_{k} \hat{R}_{k}
\end{aligned}
$$

are the system, bath, and interaction Hamiltonians, respectively. The characterization of the set of coupling constants $C_{k}$ is provided by the spectral density, which is defined as

$$
J(\omega)=\sum_{k} \frac{C_{k}^{2}}{2 m \omega_{k}} \delta\left(\omega-\omega_{k}\right) .
$$

The first master equation for this model was derived by Caldeira and Leggett [26] by using the common assumption

\footnotetext{
*matteo.carlesso@ts.infn.it

†bassi@ts.infn.it
}

of a factorized initial state

$$
\hat{\rho}_{\mathrm{T}}(0)=\hat{\rho}_{\mathrm{S}}(0) \otimes \hat{\rho}_{\mathrm{B}},
$$

where $\hat{\rho}_{\mathrm{S}}(0)$ and $\hat{\rho}_{\mathrm{B}}$ are the initial states of the system and of the bath, respectively, and also the Born-Markov approximation [2]. The high-temperature limit was taken into account in order to obtain a simple evolution [35-37] in the Lindblad form with constant coefficients

$$
\begin{aligned}
\frac{d \hat{\rho}_{\mathrm{S}}(t)}{d t}= & -\frac{i}{\hbar}\left[\hat{H}_{\mathrm{S}}, \hat{\rho}_{\mathrm{S}}(t)\right]-\frac{i \gamma}{\hbar}\left[\hat{x},\left\{\hat{p}, \hat{\rho}_{\mathrm{S}}(t)\right\}\right] \\
& -\frac{2 M \gamma}{\hbar^{2} \beta}\left[\hat{x},\left[\hat{x}, \hat{\rho}_{\mathrm{S}}(t)\right]\right],
\end{aligned}
$$

where $\gamma$ and $\beta$ are the damping rate and the inverse temperature, respectively. This derivation has two limitations. First, the master equation is the generator of a dynamical map that is not positive [38,39], i.e., it does not map all quantum states $\hat{\rho}_{\mathrm{S}}$ into quantum states. ${ }^{1}$ Second, the regime of validity cannot always be fulfilled: The latest attempts to reach the ground state in low-temperature regimes $[18,42]$ is an optomechanical example.

The main contributions in overcoming these limitations were given by Haake and Reibold [30] and later by $\mathrm{Hu}$ et al. [33], who provided the exact master equation for the particle $S$ given the total Hamiltonian $\hat{H}_{\mathrm{T}}$ :

$$
\begin{aligned}
\frac{d \hat{\rho}_{\mathrm{S}}(t)}{d t}= & -\frac{i}{\hbar}\left[\hat{H}(t), \hat{\rho}_{\mathrm{S}}(t)\right]-\frac{i \gamma(t)}{\hbar}\left[\hat{x},\left\{\hat{p}, \hat{\rho}_{\mathrm{S}}(t)\right\}\right] \\
& -h(t)\left[\hat{x},\left[\hat{x}, \hat{\rho}_{\mathrm{S}}(t)\right]\right]-f(t)\left[\hat{x},\left[\hat{p}, \hat{\rho}_{\mathrm{S}}(t)\right]\right],
\end{aligned}
$$

where $\hat{H}(t)$ and the coefficients $\gamma(t), h(t)$, and $f(t)$ now are time dependent. We refer to this model as the quantum

\footnotetext{
${ }^{1}$ Although the Caldeira-Leggett master equation (4) is not completely positive, it becomes of use when large time scales are considered. In fact, after a transient time of the order of $\sim \gamma^{-1}$, the dynamical map corresponding to Eq. (4) maps quantum states in quantum states. This is however still not sufficient to obtain in general the same asymptotic expectation values one obtains from the exact model. In order to avoid subsequent gross miscalculations, one needs to implement some effective modifications on the initial conditions $[30,40,41]$.
} 
Brownian motion (QBM) model. Contrary to the CaldeiraLeggett master equation, which is valid only for the specific ohmic choice of the spectral density $(J(\omega) \propto \omega)$, Eq. (5) is valid for arbitrary spectral densities $J(\omega)$ and temperatures $T$. However, for the QBM model, the coefficients are solutions of differential equations, which in general are hard to solve. The explicit form of these coefficients, beyond the weak-coupling limit [33], was provided by Ford and O'Connell in [43].

The generality of such a solution is outstanding; however, as noticed in [43], solving the time-dependent master equation is in general a formidable problem. Ford and O'Connell show that the dynamics of the system can be more easily solved by working with the Wigner function of the system and bath at time $t$ and then averaging over the degrees of freedom of the bath. According to their procedure, the reduced Wigner function $W$ at time $t$ can be expressed in terms of that at time $t=0$ as follows:

$$
W(x, p, t)=\int_{-\infty}^{+\infty} d r \int_{-\infty}^{+\infty} d q P(x, p ; r, q ; t) W(r, q, 0),
$$

where $P$ describes the transition probability [43].

The drawback of such a procedure is the limited selection of initial states $\hat{\rho}_{\mathrm{S}}$ for which the Wigner function is analytically computable. For Gaussian states this is not a problem; however, there exist physically relevant situations where this is not the case [44-46]. An example is provided by a system initially confined in an infinite square potential. We will refer explicitly to this example.

In this paper we propose an alternative derivation of the QBM dynamics for a general bath at arbitrary temperatures. The master equation we derive is exact and of course is equivalent to Eq. (5). However, the time-dependent coefficients will be written in a much simpler form and therefore can be used to compute much more easily the solution of the master equation, regardless of the strength of the coupling and of the form of the initial state $\hat{\rho}_{\mathrm{S}}$.

The paper is organized as follows. In Sec. II we describe our alternative derivation. In Sec. III we derive the master equation. In Sec. IV we provide a criterion for the complete positivity of the dynamics. In Sec. $\mathrm{V}$ we derive the explicit evolution of some physical quantities one is typically interested in, for a specific spectral density; in particular we compare our result with that of Haake and Reibold [30] and Hu et al. [33]. Moreover, we will show how one can easily go beyond the results of Ford and O'Connell [43] and compute the time evolution of expectations values for initial non-Gaussian states. We summarize in Sec. VI.

\section{THE QBM MODEL IN THE HEISENBERG PICTURE: THE ADJOINT MASTER EQUATION}

We derive the adjoint master equation for the quantum Brownian motion model. This is the dynamical equation describing the time evolution of a generic operator $\hat{O}$ of the system $S$, once the average over the bath is taken. To this end, we consider the unitary time evolution of the extended operator $\hat{O} \otimes \hat{\mathbf{1}}_{\mathbf{B}}$ with respect to the total Hamiltonian $\hat{H}_{\mathrm{T}}$ of the system plus bath, where $\hat{\mathbf{1}}_{\mathbf{B}}$ is the bath identity operator, and we trace over the degrees of freedom of the bath. The time derivative of the reduced operator, under the hypothesis of a factorized initial state as in Eq. (3), will be governed by the adjoint master equation.

Let us consider the von Neumann representation $[47,48]$ of the operator $\hat{O}$, defined, at time $t=0$, by the following relation:

$$
\hat{O}=\int d \lambda d \mu \mathcal{O}(\lambda, \mu) \hat{\chi}(\lambda, \mu, t=0),
$$

where $\mathcal{O}(\lambda, \mu)$ is the kernel of the operator $\hat{O}$ and $\hat{\chi}(\lambda, \mu, t=$ $0)=\exp [i \lambda \hat{x}+i \mu \hat{p}]$ is the generator of the Weyl algebra, also called characteristic or Heisenberg-Weyl operator [48]. Following the procedure previously outlined and using the von Neumann representation, the reduced operator $\hat{O}$ at time $t$ (after the trace over the bath is taken) is given by

$$
\hat{O}_{t}=\int d \lambda d \mu \mathcal{O}(\lambda, \mu) \hat{\chi}_{t},
$$

where we introduced the characteristic operator at time $t$,

$$
\hat{\chi}_{t}=\operatorname{Tr}^{(\mathrm{B})}\left(\hat{\rho}_{\mathrm{B}}\left\{\hat{\mathcal{U}}_{t}^{\dagger}\left[\hat{\chi}(\lambda, \mu, 0) \otimes \hat{\mathbf{1}}_{\mathbf{B}}\right] \hat{\mathcal{U}}_{t}\right\}\right),
$$

and $\hat{\mathcal{U}}_{t}=\exp \left(-\frac{i}{\hbar} \hat{H}_{\mathrm{T}} t\right)$. Therefore, to obtain the evolution of the operator $\hat{O}_{t}$, it is sufficient to consider the evolution equation for the characteristic operator

$$
\hat{\chi}_{t}=\operatorname{Tr}^{(\mathrm{B})}\left[\hat{\rho}_{\mathrm{B}} e^{i \lambda \hat{x}(t)+i \mu \hat{p}(t)}\right],
$$

where $\hat{x}(t)$ and $\hat{p}(t)$ are the position and momentum operators of the system $S$ evolved by the unitary evolution generated by the total Hamiltonian of the composite system plus bath and $\hat{\rho}_{\mathrm{B}}$ is defined in Eq. (3).

In order to obtain the explicit expression of $\hat{x}(t)$ and $\hat{p}(t)$, we rewrite the bath and interaction Hamiltonian defined in Eq. (1) in terms of the creation and annihilation operators $\hat{b}_{k}^{\dagger}$ and $\hat{b}_{k}$ of the $k$ th bath oscillator: $\hat{H}_{\mathrm{B}}=\sum_{k} \hbar \omega_{k} \hat{b}_{k}^{\dagger} \hat{b}_{k}$ and $\hat{H}_{\mathrm{I}}=-\hat{x} \hat{B}(0)$, where $\hat{B}(t)$ is defined as

$$
\hat{B}(t)=-\sum_{k} C_{k} \sqrt{\frac{\hbar}{2 m \omega_{k}}}\left(\hat{b}_{k} e^{-i \omega_{k} t}+\hat{b}_{k}^{\dagger} e^{i \omega_{k} t}\right) .
$$

In terms of the latter we solve the Heisenberg equations of motions for $\hat{x}(t)$ and $\hat{p}(t)$ by using the Laplace transform. The solutions are

$$
\begin{aligned}
& \hat{x}(t)=G_{1}(t) \hat{x}+G_{2}(t) \frac{\hat{p}}{M}+\frac{1}{M} \int_{0}^{t} d s G_{2}(t-s) \hat{B}(s), \\
& \hat{p}(t)=M \dot{G}_{1}(t) \hat{x}+\dot{G}_{2}(t) \hat{p}+\int_{0}^{t} d s \dot{G}_{2}(t-s) \hat{B}(s),
\end{aligned}
$$

where $\hat{x}$ and $\hat{p}$ denote the operators at time $t=0$ and the two Green's functions $G_{1}(t)$ and $G_{2}(t)$ are defined as

$$
\begin{aligned}
G_{1}(t) & =\frac{d}{d t} G_{2}(t), \\
G_{2}(t) & =\mathcal{L}^{-1}\left[\frac{M}{M\left(s^{2}+\omega_{\mathrm{R}}^{2}\right)-\mathcal{L}[D(t)](s) / \hbar}\right](t),
\end{aligned}
$$

where $\mathcal{L}$ denotes the Laplace transform and $\omega_{\mathrm{R}}^{2}=\omega_{\mathrm{S}}^{2}+$ $\frac{2}{M} \int d \omega J(\omega) / \omega$. In Eq. (13) we introduced the dissipation 
kernel $D(t)$,

$$
D(t)=2 \hbar \int_{0}^{+\infty} d \omega J(\omega) \sin (\omega t) .
$$

Given Eqs. (12), since the operators of the system and of the bath commute at the initial time, it follows that

$$
\hat{\chi}_{t}=e^{i \alpha_{1}(t) \hat{x}+i \alpha_{2}(t) \hat{p}} \operatorname{Tr}^{(\mathrm{B})}\left[\hat{\rho}_{\mathrm{B}} \hat{\chi}_{\mathrm{B}}(t)\right],
$$

where $\alpha_{1}(t)$ and $\alpha_{2}(t)$ are defined as

$$
\begin{aligned}
& \alpha_{1}(t)=\lambda G_{1}(t)+\mu M \dot{G}_{1}(t), \\
& \alpha_{2}(t)=\lambda G_{2}(t) / M+\mu \dot{G}_{2}(t)
\end{aligned}
$$

and the operator $\hat{\chi}_{\mathrm{B}}(t)$ refers only to the degrees of freedom of the bath

$$
\hat{\chi}_{\mathrm{B}}(t)=\exp \left[i \int_{0}^{t} d s \hat{B}(s) \alpha_{2}(t-s)\right] .
$$

Under the assumption of a thermal state for the bath

$$
\hat{\rho}_{\mathrm{B}} \propto e^{-\beta \hat{H}_{\mathrm{B}}},
$$

the trace over $\hat{\chi}_{\beta}(t)$ gives a real and positive function of time $\operatorname{Tr}^{(\mathrm{B})}\left[\hat{\rho}_{\mathrm{B}} \hat{\chi}_{\mathrm{B}}(t)\right]=e^{\phi(t)}$, where the explicit form of $\phi(t)$ can be obtained by using the definition of the spectral density in Eq. (2). In Appendix A we present the explicit form of $\phi(t)$, written as the sum of three terms: $\phi(t)=\lambda^{2} \phi_{1}(t)+\mu^{2} \phi_{2}(t)+$ $\lambda \mu \phi_{3}(t)$. The time derivative of $\hat{\chi}_{t}$ gives

$$
\begin{aligned}
\frac{d \hat{\chi}_{t}}{d t}= & \left(i \dot{\alpha}_{1}(t) \hat{x}+i \dot{\alpha}_{2}(t) \hat{p}+\frac{i \hbar}{2}\left[\dot{\alpha}_{1}(t) \alpha_{2}(t)\right.\right. \\
& \left.\left.-\alpha_{1}(t) \dot{\alpha}_{2}(t)\right]+\dot{\phi}(t)\right) \hat{\chi}_{t}
\end{aligned}
$$

by substituting this expression in

$$
\frac{d}{d t} \hat{O}_{t}=\int d \lambda d \mu \mathcal{O}(\lambda, \mu) \frac{d \hat{\chi}_{t}}{d t},
$$

we arrive at the adjoint master equation for the operator $\hat{O}_{t}$.

The integral in Eq. (20) depends on the choice of the kernel $\mathcal{O}(\lambda, \mu)$. On the other hand, we want an equation that can be directly applied to a generic operator $\hat{O}$ without having first to determine its kernel. This means that we want to rewrite Eq. (19) in the time-dependent form

$$
\begin{aligned}
\frac{d \hat{\chi}_{t}}{d t}= & \mathbb{L}_{t}\left[\hat{\chi}_{t}\right]=\frac{i}{\hbar}\left[\hat{H}_{\mathrm{eff}}(t), \hat{\chi}_{t}\right] \\
& +\sum_{a, b=1}^{2} K_{a b}(t)\left[\hat{L}_{a} \hat{\chi}_{t} \hat{L}_{b}^{\dagger}-\frac{1}{2}\left\{\hat{L}_{a} \hat{L}_{b}^{\dagger}, \hat{\chi}_{t}\right\}\right],
\end{aligned}
$$

where the effective Hamiltonian $\hat{H}_{\text {eff }}(t)$, the Hermitian Kossakowski matrix $\boldsymbol{K}(t)$, and the Lindblad operators $\hat{L}_{a}$ should not depend on the parameters $\lambda$ and $\mu$. Then the linearity of Eq. (20) will allow us to extend Eq. (21) to any operator $\hat{O}_{t}$. To achieve this, the explicit dependence on the parameters $\lambda$ and $\mu$, contained in the coefficients $\alpha_{i}$ and $\phi(t)$, must disappear in Eq. (19). This can be done in the following way. Let us consider the commutation relations among $\hat{x}, \hat{p}$, and $\hat{\chi}_{t}$ :

$$
\left[\hat{\chi}_{t}, \hat{x}\right]=\hbar \alpha_{2}(t) \hat{\chi}_{t}, \quad\left[\hat{\chi}_{t}, \hat{p}\right]=-\hbar \alpha_{1}(t) \hat{\chi}_{t} .
$$

Given Eqs. (16), we can express $\lambda \hat{\chi}_{t}$ and $\mu \hat{\chi}_{t}$ as a linear combination of the above commutators. Then, by using this result, we can easily rewrite Eq. (19) in the form given by Eq. (21), where

$$
\hat{H}_{\mathrm{eff}}(t)=\frac{\hat{p}^{2}}{2 M}+\frac{\Gamma^{\mathrm{A}}(t)}{2}(\hat{x} \hat{p}+\hat{p} \hat{x})+\frac{1}{2} M \Delta^{\mathrm{A}}(t) \hat{x}^{2}
$$

and the Lindblad operators are $\hat{L}_{1}=\hat{x}$ and $\hat{L}_{2}=\hat{p}$. The time-dependent function $\Gamma^{\mathrm{A}}(t), \Delta^{\mathrm{A}}(t)$, and the elements of the Kossakowski matrix $K_{a, b}(t)$ are reported in Appendix B. It is important to note that one of the elements of the Kossakowski matrix vanishes: $K_{22}(t)=0$. This means that the term corresponding to $\left[\hat{p},\left[\hat{p}, \hat{\rho}_{\mathrm{s}}\right]\right]$ is absent, as for the Caldeira-Leggett master equation [26]. In the latter case this implies the noncomplete positivity of the dynamics. In the case under study, complete positivity is instead automatically satisfied, as it is explicitly shown in Sec. IV. This result is in agreement with previous results $[30,33,43,49]$.

Equation (21) is linear in $\hat{\chi}_{t}$ and does not depend on $\lambda$ and $\mu$. Therefore, because of Eq. (20), it holds for any operator $\hat{O}_{t}, \frac{d}{d t} \hat{O}_{t}=\mathbb{L}_{t}\left[\hat{O}_{t}\right]$, as in Eq. (21). This is the adjoint master equation and $\mathbb{L}_{t}$ is the generator of the dynamics. The correspondent adjoint dynamical map is given by

$$
\boldsymbol{\Phi}_{t}[\cdot]=\mathcal{T} \exp \left(\int_{0}^{t} d s \mathbb{L}_{s}\right)[\cdot] .
$$

The result obtained here is very general and depends only on the form of the total Hamiltonian $\hat{H}_{\mathrm{T}}$ defining the QBM model together with the separability of the initial total state (3), but does not depend on the particular initial state of the system $S$. We now show that we recover the master equation (5) for the states.

\section{MASTER EQUATION FOR THE STATISTICAL OPERATOR}

We now derive the master equation for the density matrix, starting from the adjoint master equation. For a time-independent adjoint master equation, switching to the master equation for the states is straightforward: The adjoint dynamical map $\boldsymbol{\Phi}_{t}$ is $\exp (t \mathbb{L})$, where the generator $\mathbb{L}$ is time independent. Therefore, the map $\boldsymbol{\Phi}_{t}$ and its generator $\mathbb{L}$ commute. Then the generator of the dynamics for the states is equal to the adjoint of the generator of the dynamics for the operators. In the time-dependent case here considered, instead the procedure is more delicate. Consider the dynamical map $\boldsymbol{\Phi}_{t}^{*}$ for the states:

$$
\boldsymbol{\Phi}_{t}^{*}: \hat{\rho}_{\mathrm{S}}(0) \mapsto \hat{\rho}_{\mathrm{S}}(t),
$$

which is the adjoint map of $\boldsymbol{\Phi}_{t}$ defined in Eq. (24). The adjointness, denoted here by the asterisk, has to be understood in the following sense:

$$
\left\langle\hat{\chi}_{t}\right\rangle=\operatorname{Tr}^{\mathrm{S}}\left\{\boldsymbol{\Phi}_{t}[\hat{\chi}(0)] \hat{\rho}_{\mathrm{S}}(0)\right\}=\operatorname{Tr}^{\mathrm{S}}\left\{\hat{\chi}(0) \boldsymbol{\Phi}_{t}^{*}\left[\hat{\rho}_{\mathrm{S}}(0)\right]\right\},
$$

where $\langle\cdot\rangle=\operatorname{Tr}^{\mathrm{S}}\left\{\cdot \hat{\rho}_{\mathrm{S}}(0)\right\}$. Let us consider the time derivative of $\left\langle\hat{\chi}_{t}\right\rangle$ and let us express it as follows:

$$
\frac{d}{d t}\left\langle\hat{\chi}_{t}\right\rangle=\operatorname{Tr}^{\mathrm{S}}\left\{\boldsymbol{\Lambda}_{t}[\hat{\chi}(0)] \hat{\rho}_{\mathrm{S}}(0)\right\}=\operatorname{Tr}^{\mathrm{S}}\left\{\hat{\chi}(0) \boldsymbol{\Lambda}_{t}^{*}\left[\hat{\rho}_{\mathrm{S}}(0)\right]\right\} .
$$


This defines the two maps $\boldsymbol{\Lambda}_{t}$ and $\boldsymbol{\Lambda}_{t}^{*}$. According to Eq. (21),

$$
\boldsymbol{\Lambda}_{t}[\hat{\chi}(0)]=\mathbb{L}_{t}\left[\hat{\chi}_{t}\right]=\mathbb{L}_{t} \circ \boldsymbol{\Phi}_{t}[\hat{\chi}(0)] .
$$

On the other hand, according to standard practice [2], the map $\boldsymbol{\Lambda}_{t}^{*}$ in Eq. (27) is defined as

$$
\boldsymbol{\Lambda}_{t}^{*}\left[\hat{\rho}_{\mathrm{S}}(0)\right]=\tilde{\mathbb{L}}_{t}^{*}\left[\hat{\rho}_{\mathrm{S}}(t)\right]=\tilde{\mathbb{L}}_{t}^{*} \circ \boldsymbol{\Phi}_{t}^{*}\left[\hat{\rho}_{\mathrm{S}}(0)\right],
$$

where the map $\tilde{\mathbb{L}}_{t}^{*}$ is the generator of the dynamics for the states. By adjointness we have

$$
\operatorname{Tr}^{\mathrm{S}}\left\{\mathbb{L}_{t} \circ \boldsymbol{\Phi}_{t}[\hat{\chi}(0)] \hat{\rho}_{\mathrm{S}}(0)\right\}=\operatorname{Tr}^{\mathrm{S}}\left\{\boldsymbol{\Phi}_{t} \circ \tilde{\mathbb{L}}_{t}[\hat{\chi}(0)] \hat{\rho}_{\mathrm{S}}(0)\right\} .
$$

Then, by comparison, we have to construct the map $\tilde{\mathbb{L}}_{t}$ as follows:

$$
\tilde{\mathbb{L}}_{t}=\boldsymbol{\Phi}_{t}{ }^{-1} \circ \mathbb{L}_{t} \circ \boldsymbol{\Phi}_{t} .
$$

In terms of this latter expression, Eq. (28) becomes

$$
\boldsymbol{\Lambda}_{t}[\hat{\chi}(0)]=\boldsymbol{\Phi}_{t} \circ \tilde{\mathbb{L}}_{t}[\hat{\chi}(0)] .
$$

For a time-dependent generator, in order to construct the master equation for the states we need to derive explicitly the form of $\tilde{\mathbb{L}}_{t}$. This is derived in Appendix $\mathrm{C}$ and the final result is

$$
\begin{aligned}
\tilde{\mathbb{L}}_{t}[\hat{\chi}(0)]= & \frac{i}{\hbar}\left[\hat{\tilde{H}}_{\mathrm{eff}}(t), \hat{\chi}(0)\right] \\
& +\sum_{a, b=1}^{2} \tilde{K}_{a b}(t)\left[\hat{L}_{a} \hat{\chi}(0) \hat{L}_{b}^{\dagger}-\frac{1}{2}\left\{\hat{L}_{a} \hat{L}_{b}^{\dagger}, \hat{\chi}(0)\right\}\right]
\end{aligned}
$$

where $\hat{L}_{\alpha}$ is defined after Eq. (23),

$$
\hat{\tilde{H}}_{\mathrm{eff}}(t)=\frac{\hat{p}^{2}}{2 M}-\frac{\Gamma^{\mathrm{A}}(t)}{2}(\hat{x} \hat{p}+\hat{p} \hat{x})+\frac{1}{2} M \Delta^{\mathrm{A}}(t) \hat{x}^{2},
$$

and the elements of $\tilde{K}_{a b}(t)$ are reported in Appendix C. Now, in order to obtain the time derivative of the operator $\hat{O}_{t}$ at time $t$, we act with $\tilde{\mathbb{L}}_{t}$ on the operator $\hat{O}(0)$ at time $t=0$ and then with the adjoint dynamical map $\boldsymbol{\Phi}_{t}$, as described in Eq. (32). The latter equation, according to the definition of the map $\boldsymbol{\Lambda}_{t}$ in Eq. (28), gives $\frac{d}{d t} \hat{O}_{t}$. From this we can compute the master equation for the states. This can be simply done by using the cyclic property of the trace $\operatorname{Tr}^{(\mathrm{S})}[\cdot]$ applied on the expression (33). Then we have

$$
\frac{d}{d t}\left\langle\hat{\chi}_{t}\right\rangle=\operatorname{Tr}^{\mathrm{S}}\left\{\tilde{\mathbb{L}}_{t}[\hat{\chi}(0)] \boldsymbol{\Phi}_{t}^{*}\left[\hat{\rho}_{\mathrm{S}}(0)\right]\right\}=\operatorname{Tr}^{\mathrm{S}}\left(\hat{\chi}(0) \frac{d}{d t} \hat{\rho}_{\mathrm{S}}(t)\right)
$$

which yields the master equation for the states of the system $S$,

$$
\begin{aligned}
\frac{d \hat{\rho}_{\mathrm{S}}(t)}{d t}= & -\frac{i}{\hbar}\left[\hat{\tilde{H}}_{\mathrm{eff}}(t), \hat{\rho}_{\mathrm{S}}(t)\right] \\
& +\sum_{a, b=1}^{2} \tilde{K}_{a b}(t)\left[\hat{L}_{b}^{\dagger} \hat{\rho}_{\mathrm{S}}(t) \hat{L}_{a}-\frac{1}{2}\left\{\hat{L}_{a} \hat{L}_{b}^{\dagger}, \hat{\rho}_{\mathrm{S}}(t)\right\}\right],
\end{aligned}
$$

This is the desired result, which naturally coincides with the QBM master equation (5). The explicit form of the terms in Eq. (36) can be obtained starting from the spectral density $J(\omega)$ defined in Eq. (2).

\section{COMPLETE POSITIVITY}

We now discuss the complete positivity of the dynamical map $\boldsymbol{\Phi}_{t}$ generated by the generator $\mathbb{L}_{t}$ defined in Eq. (21). The action of this dynamical map on the generic operator $\hat{O}$ of the system $S$ is

$$
\boldsymbol{\Phi}_{t}[\hat{O}]=\hat{O}_{t}=\operatorname{Tr}^{\mathrm{B}}\left\{\hat{\rho}_{\mathrm{B}}\left[\hat{\mathcal{U}}_{t}^{\dagger}\left(\hat{O} \otimes \hat{\mathbf{1}}_{\mathbf{B}}\right) \hat{\mathcal{U}}_{t}\right]\right\}
$$

which is the combination of two completely positive maps: the unitary evolution provided by the total Hamiltonian of system plus bath and the trace over the bath. Therefore, by construction, the dynamical map is completely positive. However, two observations are relevant here. First, it is instructive to verify explicitly the complete positivity of the dynamics. Second, in a situation where approximations are needed in order to compute explicitly the coefficients of the (adjoint) master equation, the verification of the complete positivity of the dynamics becomes a fundamental point of interest.

When the generator $\mathbb{L}$ of the dynamics is not time dependent, the sufficient and necessary condition for the complete positivity of the dynamical map is the positivity of the Kossakowski matrix [36,50]. For a time-dependent generator $\mathbb{L}_{t}$, instead a positive Kossakowski matrix is only a sufficient condition for complete positivity. An example is precisely the QBM model under study, whose Kossakowski matrix is not positive for all times; nevertheless, the dynamics is completely positive. For a time-dependent generator, a necessary and sufficient condition instead is given by the following theorem [51,52], under the assumption of a Gaussian channel.

Suppose that the action of a Gaussian dynamical map $\boldsymbol{\Phi}_{t}$ on the characteristic operator $\hat{\chi}$ of the system is defined as

$$
\boldsymbol{\Phi}_{t}: \exp (i\langle\xi \mid R\rangle) \mapsto \exp \left(i\left\langle\xi\left|\boldsymbol{X}_{t}\right| R\right\rangle\right) \exp \left(-\frac{1}{2}\left\langle\xi\left|\boldsymbol{Y}_{t}\right| \xi\right\rangle\right),
$$

where $\boldsymbol{X}_{t}$ and $\boldsymbol{Y}_{t}$ are $2 \times 2$ matrices describing the evolution of the characteristic operator

$$
\begin{aligned}
\boldsymbol{X}_{t} & =\left(\begin{array}{cc}
G_{1}(t) & G_{2}(t) / M \\
M \dot{G}_{1}(t) & \dot{G}_{2}(t)
\end{array}\right), \\
\boldsymbol{Y}_{t} & =\left(\begin{array}{cc}
-2 \phi_{1}(t) & -\phi_{3}(t) \\
-\phi_{3}(t) & -2 \phi_{2}(t)
\end{array}\right),
\end{aligned}
$$

and $\langle\xi|=(\lambda, \mu)$ and $\langle R|=(\hat{x}, \hat{p})$. In terms of $\boldsymbol{X}_{t}, \boldsymbol{Y}_{t}$, and the symplectic matrix $\boldsymbol{\Omega}=\left(\begin{array}{cc}0 & 1 \\ -1 & 0\end{array}\right)$, we can define the following matrix $\boldsymbol{\Psi}_{t}$ :

$$
\boldsymbol{\Psi}_{t}=\boldsymbol{Y}_{t}+\frac{i \hbar}{2} \boldsymbol{\Omega}-\frac{i \hbar}{2} \boldsymbol{X}_{t} \boldsymbol{\Omega} \boldsymbol{X}_{t}^{\top}
$$

The necessary and sufficient condition for the dynamical map $\boldsymbol{\Phi}_{t}$ to be completely positive (CP) is the positivity of $\boldsymbol{\Psi}_{t}$ for all positive times. Since the matrix $\boldsymbol{\Psi}_{t}$ is a $2 \times 2$ matrix, the request of its positivity reduces to the request of positivity of its trace and determinant:

$$
\begin{aligned}
\operatorname{Tr}\left[\boldsymbol{\Psi}_{t}\right] & =-2\left[\phi_{1}(t)+\phi_{2}(t)\right], \\
\operatorname{det}\left[\boldsymbol{\Psi}_{t}\right] & =4 \phi_{1}(t) \phi_{2}(t)-\phi_{3}^{2}(t)-\frac{1}{4}[\hbar-F(t)]^{2} .
\end{aligned}
$$


The condition of positivity of the trace [Eq. (41a)] is easily verified for all physical spectral densities: The spectral density is positive by definition [see Eq. (2)] and this implies the negativity of $\phi_{1}(t)$ and $\phi_{2}(t)$ [see Eqs. (A1)] for all positive values of the temperature. On the other hand, the second condition (41b) cannot be easily verified in general. Once a specific spectral density $J(\omega)$ is chosen, one can check explicitly whether $\operatorname{det}\left[\boldsymbol{\Psi}_{t}\right] \geqslant 0$. For example, the spectral density $J(\omega) \propto \omega$, originally chosen in [26] to describe the quantum Brownian motion, does not satisfy the above condition also in the case of no external potentials and in fact it is well known that the Caldeira-Leggett master equation is not $\mathrm{CP}$.

As already remarked, the QBM model automatically guarantees complete positivity. However, in practical cases one is not able to compute explicitly the time-dependent coefficients of the Kossakowski matrix. Approximations are needed, in which case complete positivity is not automatically guaranteed anymore. This can be checked in a relatively easy way by assessing the positivity of $\operatorname{det}\left[\boldsymbol{\Psi}_{t}\right]$.

\section{TIME EVOLUTION OF RELEVANT QUANTITIES}

The original QBM master equation (5) is expressed in terms of functions (forming the Kossakowski matrix), whose explicit expression is not easy to derive, even if one considers the solution given in [43]. They are solutions of complicated differential equations, difficult to solve except for very simple situations. More importantly, expectation values are not easy to compute: One has to determine the state of the system at time $t$, which is in general a formidable problem also in a particularly simple situation. In our derivation, instead the use of the adjoint master equation provides a much easier tool for the computation of expectation values. The evolution is expressed in the Heisenberg picture; therefore, it does not depend on the state of the system $S$, but only on the properties of the adjoint evolution $\boldsymbol{\Phi}_{t}$.

For example, by plugging the expression of $\hat{x}^{2}(t)$ [obtained from Eq. (12)] in Eq. (21) we obtain an equation for the expectation value $\left\langle\hat{x}_{t}^{2}\right\rangle$,

$$
\begin{aligned}
\frac{d}{d t}\left\langle\hat{x}_{t}^{2}\right\rangle= & 2 \dot{G}_{1}(t) \dot{G}_{2}(t)\left\langle\hat{x}^{2}\right\rangle+2 \dot{G}_{1}(t) \dot{G}_{2}(t)\left\langle\hat{p}^{2}\right\rangle / M^{2} \\
& +\left[G_{1}(t) \dot{G}_{2}(t)+\dot{G}_{1}(t) G_{2}(t)\right] \\
& \times\langle\{\hat{x}, \hat{p}\}\rangle / M-2 \dot{\phi}_{1}(t),
\end{aligned}
$$

which can be solved directly without having to solve a more complicated system of differential equations, as it is necessary when the solution is in the Schrödinger picture [2], as well as for the case of the Wigner function approach $[43,53,54]$. Once the interaction with the bath, i.e., the spectral density function, is specified, $G_{1}(t)$ and $G_{2}(t)$ can be determined as described before and this fully determines the time evolution of $\left\langle\hat{x}_{t}^{2}\right\rangle$ in terms of the initial expectation values. In a similar way one can compute all the other expectation values as a function of time.

To show this, we provide the explicit general solution of some physical quantities of interest for a specific spectral density. We consider the diffusion function $\Lambda^{\mathrm{dif}}(t)=\left\langle\hat{x}_{t}^{2}\right\rangle-\left\langle\hat{x}_{t}\right\rangle^{2}$, the energy of the system $E(t)=\left\langle\hat{p}_{t}^{2}\right\rangle / 2 M+\frac{1}{2} M \omega_{\mathrm{S}}^{2}\left\langle\hat{x}_{t}^{2}\right\rangle$, and the decoherence function $\Gamma_{\mathrm{dec}}(t)$. The latter is defined as follows. We consider a particle that, at time $t=0$, is described by a state $|\psi(t=0)\rangle=\mathcal{N}[|\alpha\rangle+|\beta\rangle]$, where $|\alpha\rangle$ and $|\beta\rangle$ are two equally spread out, with spread equal to $\sigma_{0}$, Gaussian wave packets, centered, respectively, in $x_{\alpha}=\langle\alpha|\hat{x}| \alpha\rangle$ and $x_{\beta}=\langle\beta|\hat{x}| \beta\rangle$, and $\mathcal{N}$ is the normalization constant. The probability density in position $x$ at time $t$ is [2]

$$
\begin{aligned}
\mathcal{P}(x, t)= & \mathcal{N}^{2}\left\{\rho_{\alpha \alpha}(x, t)+\rho_{\beta \beta}(x, t)\right. \\
& \left.+2 \sqrt{\rho_{\alpha \alpha}(x, t) \rho_{\beta \beta}(x, t)} \exp \left[\Gamma^{\operatorname{dec}}(t)\right] \cos [\varphi(x, t)]\right\},
\end{aligned}
$$

where $\rho_{\alpha \beta}(x, t)=\left\langle x\left|\operatorname{Tr}^{(\mathrm{B})}\left[\mathcal{U}_{t}(|\alpha\rangle\langle\beta|) \mathcal{U}_{t}^{\dagger}\right]\right| x\right\rangle$ : There is a modulation given by the phase $\varphi(x, t)$ and a reduction of the interference contrast determined by the decoherence function $\Gamma^{\operatorname{dec}}(t)<0$. The decoherence function takes the following form:

$$
\begin{aligned}
\Gamma^{\operatorname{dec}}(t)= & -\frac{4 \sigma_{0}^{4} \Delta_{p}^{2}+\hbar^{2} \Delta_{x}^{2}}{\hbar^{2}} \\
& \times \frac{M^{2} \phi_{1}(t)}{8 M^{2} \sigma_{0}^{2} \phi_{1}(t)-\hbar^{2} G_{2}^{2}(t)-4 M^{2} \sigma_{0}^{4} G_{1}^{2}(t)},
\end{aligned}
$$

where $\Delta_{x}$ and $\Delta_{p}$ are the distances between the two Gaussian states in position and momentum and the function $\phi_{1}(t)$ is defined in Eq. (A1a). The explicit expressions for $\Lambda^{\operatorname{dif}}(t)$ and $E(t)$ are given in Appendix D.

As a concrete example, we consider the case of the DrudeLorentz spectral density

$$
J(\omega)=\frac{2}{\pi} M \gamma \Omega^{2} \frac{\omega}{\omega^{2}+\Omega^{2}},
$$

which is commonly used for example in light-harvesting systems [13,55], where $\Omega$ is the characteristic frequency of the bath. The corresponding dissipation and noise kernels, defined in Eqs. (14) and (A2), respectively, are

$$
\begin{aligned}
D(t)= & 2 M \gamma \hbar \Omega^{2} e^{-\Omega|t|} \operatorname{sgn}(t), \\
D_{1}(t)= & \frac{2 M \gamma \hbar \Omega^{2}}{\pi}\left[\Phi_{L}\left(e^{-2 \pi|t| / \beta \hbar}, 1,-\frac{\beta \hbar \Omega}{2 \pi}\right)\right. \\
& +\Phi_{L}\left(e^{-2 \pi|t| / \beta \hbar}, 1, \frac{\beta \hbar \Omega}{2 \pi}\right) \\
& \left.+\pi e^{-\Omega|t|} \cot \left(\frac{\beta \hbar \Omega}{2}\right)\right],
\end{aligned}
$$

where the function $\Phi_{L}$ is the Hurwitz-Lerch transcendent function $\Phi_{L}(z, s, a)=\sum_{n=0}^{+\infty} z^{n}(n+a)^{-s}$. The two Green's functions are

$$
G_{2}(t)=\sum_{i=1}^{3} \frac{\left(\Omega+C_{i}\right) e^{C_{i} t}}{D_{i}}
$$

and $G_{1}(t)=\frac{d}{d t} G_{2}(t)$, where $C_{1}, C_{2}$, and $C_{3}$ are the complex roots of the polynomial $y(s)=\left(y^{2}+\omega_{\mathrm{S}}^{2}+2 \gamma \Omega\right)(y+$ $\Omega)-2 \gamma \Omega^{2}$ and $D_{i}=\prod_{j=1, j \neq i}^{3}\left(C_{i}-C_{j}\right)$. In terms of these functions, we can compute the functions $\phi_{i}(t)$ with the help of Eqs. (A1) as well as the three relevant quantities previously discussed, whose explicit expressions are displayed in Eqs. (44), (D3), and (D4). 

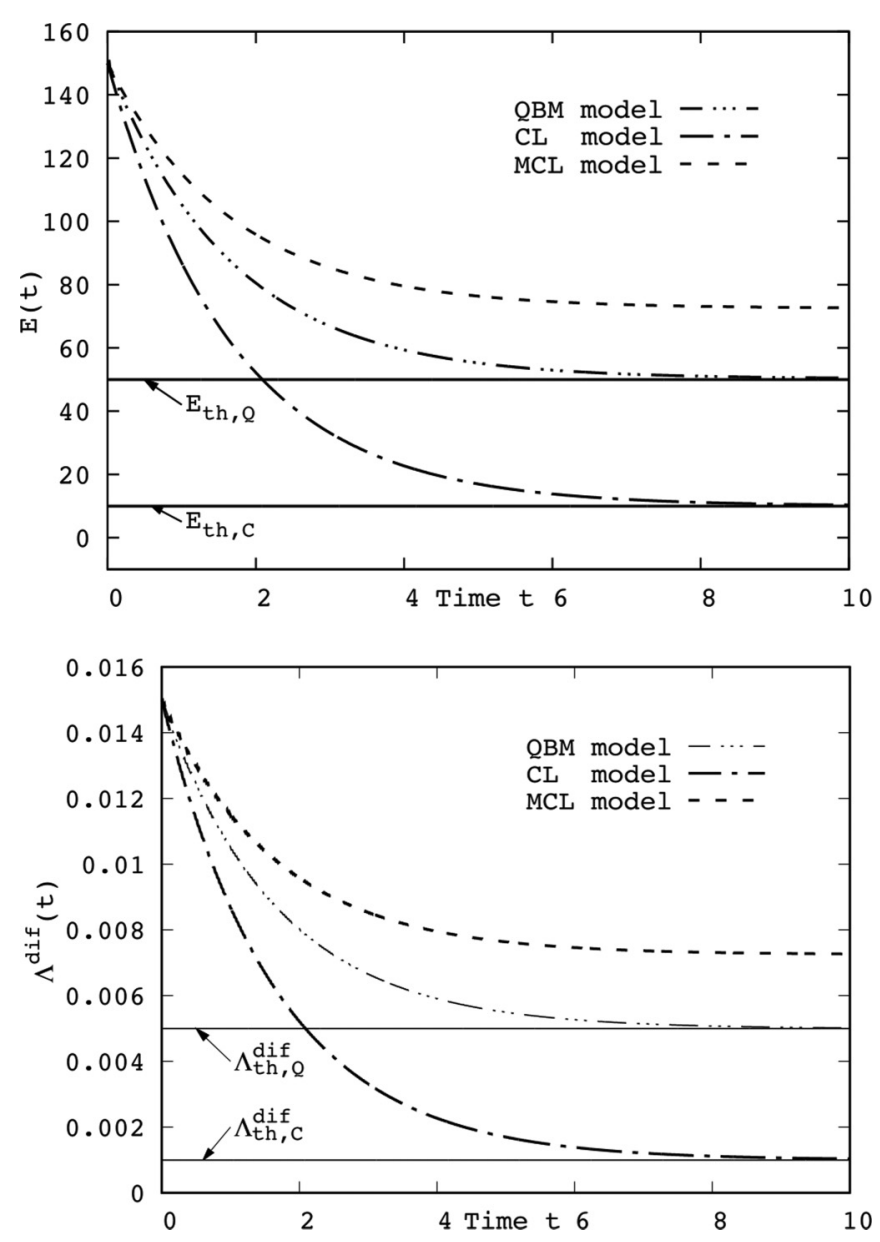

FIG. 1. Time evolution of the energy $E(t)$ (top panel) and diffusion in space $\Lambda^{\mathrm{dif}}(t)$ (bottom panel) for the first excited state of the harmonic oscillator with frequency $\omega_{\mathrm{S}}=100$ centered in the origin $(\langle\hat{x}\rangle=0=\langle\hat{p}\rangle)$ with the parameters $M=1, \gamma=0.3$, $\Omega=2000, \beta=10^{-1}$, and $\hbar=1$. The plot shows the behavior of $E(t)$ and $\Lambda^{\mathrm{dif}}(t)$ for the QBM model with the Drude-Lorentz spectral density, for the Caldeira-Leggett model (CL), and for its modification (MCL). In addition, $E_{\mathrm{th}, \mathrm{Q}}, E_{\mathrm{th}, \mathrm{C}}, \Lambda_{\mathrm{th}, \mathrm{Q}}^{\mathrm{dif}}$, and $\Lambda_{\mathrm{th}, \mathrm{C}}^{\mathrm{dif}}($ see the main text) are also plotted.

Figures 1 and 2 show the evolution of the diffusion function $\Lambda^{\mathrm{dif}}(t)$, of the energy $E(t)$, and of the decoherence function $\Gamma^{\operatorname{dec}}(t)$ and we compare their time evolution according to the QBM model as described above with that of the CaldeiraLeggett (CL) master equation in (4). We also consider the evolution given by the modification of the Caldeira-Leggett (MCL) master equation, which is obtained from Eq. (4) by adding the term $-\frac{\gamma \beta}{8 M}\left[\hat{p},\left[\hat{p}, \hat{\rho}_{\mathrm{S}}(t)\right]\right]$ to guarantee the complete positivity of the dynamics $[2,38,39]$. As for the initial state, in Fig. 1 we considered the first excited state of the harmonic oscillator with frequency $\omega_{\mathrm{S}}$ centered at the origin: $\langle\hat{x}\rangle=0=\langle\hat{p}\rangle$.

The asymptotic value of $E(t)$ is given by the equilibrium energy of the thermal state $\hat{\rho}_{t h} \propto \exp \left(-\beta H_{\mathrm{S}}\right)$ :

$$
E_{\mathrm{th}, \mathrm{Q}}=\frac{\hbar \omega_{\mathrm{S}}}{2}+\frac{\hbar \omega_{\mathrm{S}}}{e^{\beta \hbar \omega_{\mathrm{S}}}-1}
$$

which in the high-temperature limit coincides with the classical value $E_{\mathrm{th}, \mathrm{C}}=1 / \beta$. For high temperatures the difference between the two thermal energies $E_{\mathrm{th}, \mathrm{Q}}$ and $E_{\mathrm{th}, \mathrm{C}}$ is negligible; in this case the three dynamics lead to the same asymptotic value. This is expected since both the CL and MCL master equations are derived in the high-temperature limit and our result is exact. However, at low temperatures, as Fig. 1 shows, the difference between the quantum and classical cases becomes important and shows the quantum properties of the system $S$ : The zero-point energy $\hbar \omega_{\mathrm{S}} / 2$ is the minimal allowed energy. The CL dynamics, at low temperatures, fails to capture this feature since its asymptotic value is lower. The MCL dynamics leads to an asymptotic energy that is different from both the classical and the quantum values. This is due to the correction to the Caldeira-Leggett master equation. As mentioned before, the latter is needed to satisfy complete positivity; however, it leads to unphysical effects, e.g., the system is overheated. Only the QBM model displays the correct quantum behavior.

A similar situation is found for the diffusion in position $\Lambda^{\mathrm{dif}}(t)$. According to the well-known result of equilibrium quantum statistical physics, its asymptotic value is given by $[26,56]$

$$
\Lambda_{\mathrm{th}, \mathrm{Q}}^{\mathrm{dif}}=\frac{\hbar}{2 M \omega_{\mathrm{S}}} \operatorname{coth}\left(\frac{\beta \hbar \omega_{\mathrm{S}}}{2}\right),
$$

which is the diffusion for a harmonic oscillator in the thermal state $\hat{\rho}_{\text {th }}$. In the high-temperature limit (49) gives the classical asymptotic value $\Lambda_{\mathrm{th}, \mathrm{C}}^{\mathrm{dif}}=1 / M \beta \omega_{\mathrm{S}}^{2}$. Again, for high temperatures the difference between the classical and the quantum thermal diffusion can be neglected and the three dynamics give the same result. For low temperatures the difference becomes important. The MCL asymptotic value differs from both the classical and quantum equilibrium values.

Figure 2 shows how $\Gamma^{\mathrm{dec}}(t)$ decays in time. For high temperatures, $\exp \left[\Gamma^{\operatorname{dec}}(t)\right]$ reaches rapidly its asymptotic value, i.e.,

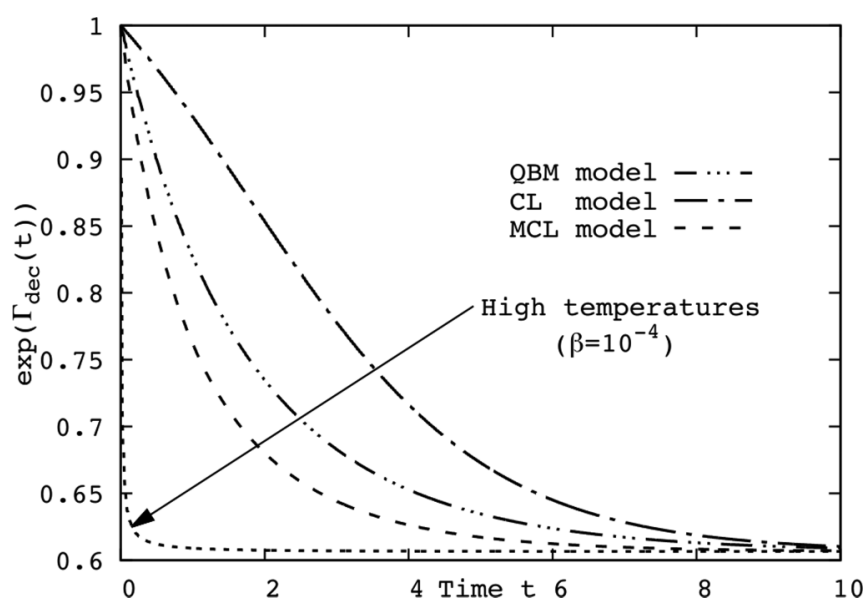

FIG. 2. Decoherence function $\exp \left[\Gamma^{\mathrm{dec}}(t)\right]$ with the parameters $M=1, \gamma=0.3, \Omega=200, \omega_{\mathrm{S}}=100, \hbar=1, \sigma_{0}=\sqrt{\hbar / 2 M \omega_{\mathrm{S}}}$, $\Delta_{x}=2 \sigma_{0}$, and $\Delta_{p}=0$. The plot shows the behavior of the decoherence function $\exp [\Gamma(t)]$ for the Drude-Lorentz spectral density (QBM), for the Caldeira-Leggett model (CL), and for its modification (MCL) at two different temperatures: $\beta=10^{-1}$ and $\beta=10^{-4}$. For $\beta=10^{-4}$ the differences between the three models are minimal and the three curves coincide with the dotted line. 
the decoherence time $\tau_{\mathrm{D}}$ is very short. In the low-temperature case instead $\tau_{\mathrm{D}}$ is higher. Notice that the asymptotic value in both cases is not zero but, in agreement with the literature [2], it saturates at a finite value:

$$
\Gamma^{\mathrm{dec}}(\infty)=-\frac{1}{8} \frac{\Delta_{x}^{2}}{\sigma_{0}^{2}} .
$$

Again, there are differences between the three dynamics. In particular, with respect to the QBM result, the CL dynamics overestimates the decoherence time $\tau_{\mathrm{D}}$, whereas for the MCL dynamics it is underestimated.

\section{Non-Gaussian initial state}

The following example will make clear the advantage of the present approach. Consider a system initially confined by the square potential $V(x)=0$ for $x \in[0, a]$ and $V(x)=+\infty$ otherwise, at rest in the ground state. The system later evolves subject to the harmonic potential. The initial state then is

$$
\psi(x)= \begin{cases}\sqrt{2 / a} \sin (\pi x / a) & \text { for } x \in[0, a] \\ 0 & \text { otherwise. }\end{cases}
$$

The corresponding initial expectation values for the quadratic operators are

$$
\begin{aligned}
\langle\hat{x}\rangle & =\frac{a}{2}, \quad\langle\hat{p}\rangle=0, \quad\left\langle\hat{x}^{2}\right\rangle=\frac{1}{6}\left(2-\frac{3}{\pi^{2}}\right) a^{2}, \\
\left\langle\hat{p}^{2}\right\rangle & =\frac{\pi^{2} \hbar^{2}}{a^{2}}, \quad\langle\{\hat{x}, \hat{p}\}\rangle=0 .
\end{aligned}
$$

The time evolution of the diffusion function $\Lambda^{\operatorname{dif}}(t)$ and energy $E(t)$ is easy to obtain, as one can see from Eqs. (D3) and (D4). In fact, in our approach the only quantities that might change, when changing the state of the system, are the initial expectation values. The functional dependence of the physical quantities on the initial values instead does not change. Then, by plugging in Eqs. (D3) and (D4) the initial expectation values for the non-Gaussian state (52), one directly obtains the time evolution of $\Lambda^{\mathrm{dif}}(t)$ and $E(t)$, which are plotted ${ }^{2}$ in Fig. 3. While the time evolution of $E(t)$ is qualitatively the same as in the example previously considered, the diffusion function $\Lambda^{\text {dif }}(t)$ shows high-frequency oscillations when the initial state is taken equal to Eq. (51). These oscillations arise from the choice of the initial state and are present also when the system is isolated.

With no bath, the diffusion function is equal to

$$
\begin{aligned}
\Lambda^{\operatorname{dif}}(t)= & \cos ^{2} \omega_{\mathrm{S}} t\left(\left\langle\hat{x}^{2}\right\rangle-\langle\hat{x}\rangle^{2}\right)+\frac{\sin ^{2} \omega_{\mathrm{S}} t}{M^{2} \omega_{\mathrm{S}}^{2}}\left(\left\langle\hat{p}^{2}\right\rangle-\langle\hat{p}\rangle^{2}\right) \\
& +\frac{2 \cos \omega_{\mathrm{S}} t \sin \omega_{\mathrm{S}} t}{M \omega_{\mathrm{S}}}\left(\frac{\langle\{\hat{x}, \hat{p}\}\rangle}{2}-\langle\hat{x}\rangle\langle\hat{p}\rangle\right) .
\end{aligned}
$$

\footnotetext{
${ }^{2}$ The ground state of the square potential was preferred to its first excited since the initial values of the energy and diffusion function are more compatible with those of the first excited state of the harmonic oscillator. However, a similar time dependence is shown when the initial state is taken to be equal to the first excited state of the square potential.
}
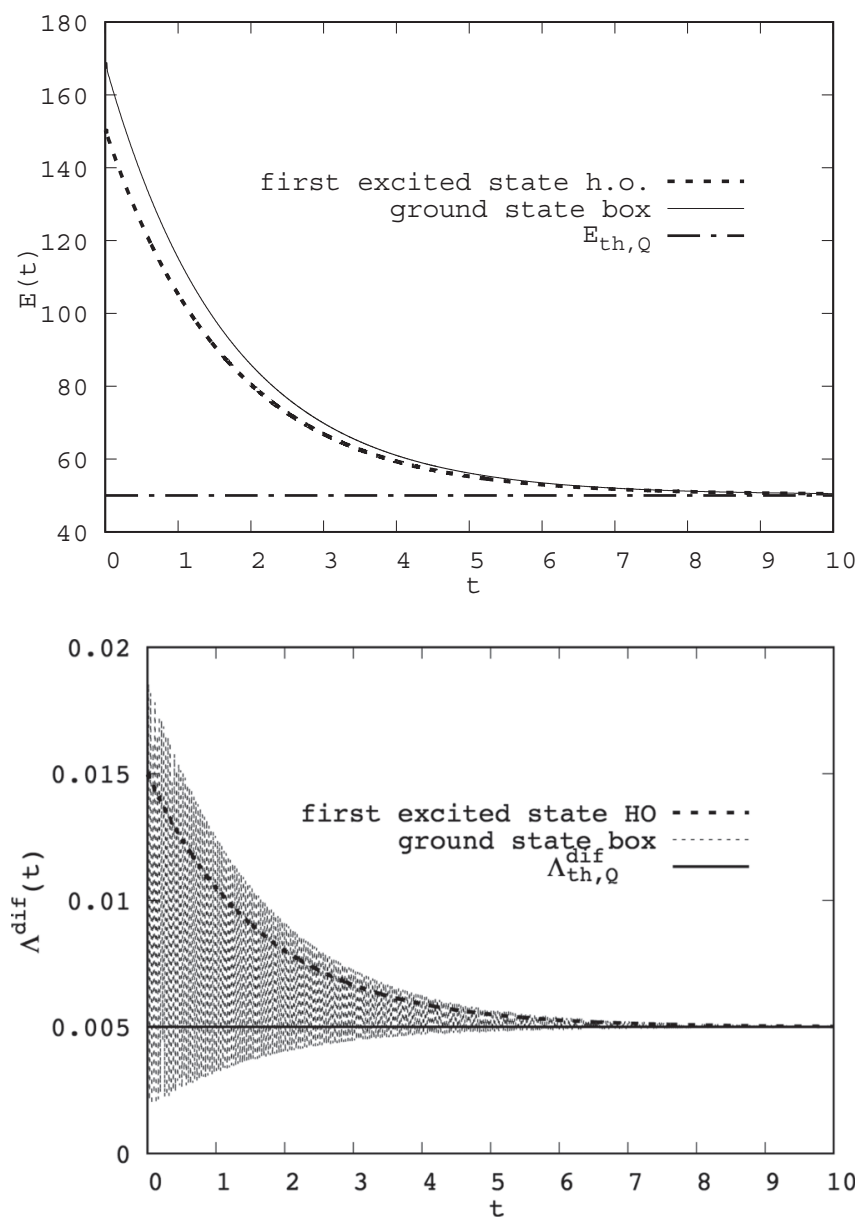

FIG. 3. Comparison of the solutions of the QBM model for two different initial states: the first excited state of the harmonic oscillator $(\mathrm{HO})$ at frequency $\omega_{\mathrm{S}}$ (dashed line) and the ground state of the square potential displayed in Eq. (51) (solid line). The chosen parameters are $M=1, \gamma=0.3, \Omega=2000, \omega_{\mathrm{S}}=100, \beta=10^{-1}, a=0.23$, and $\hbar=1$. The top panel shows the evolution of the energy $E(t)$ for the two systems, compared with the equilibrium energy $E_{\mathrm{th}, \mathrm{Q}}$ for the quantum thermal state. The bottom panel shows the evolution of the diffusion function $\Lambda^{\text {diff }}(t)$ for the two systems, compared with the equilibrium diffusion $\Lambda_{\mathrm{th}, \mathrm{Q}}^{\mathrm{diff}}$ for the quantum thermal state.

By plugging into this expression the expectation values for the ground state of the square potential [see Eq. (52)] we obtain the oscillatory behavior, while for the eigenstates of the harmonic oscillator (53) the diffusion of course is constant [and correspondingly when the bath is switched on, $\Lambda^{\operatorname{dif}}(t)$ simply decays exponentially as plotted in Fig. 3].

As we have shown, the evolution of the expectation values is easy to obtain by using our approach. Once the functional dependence of the physical quantities on the initial values is computed, we direct obtain their time dependence for different initial states simply by inserting the initial expectation values. On the other hand, when working in the Schrödinger picture, as typically done in the literature [2,33], or with the Wigner formalism $[43,53,54]$, one has to find the explicit time evolution of the initial state, which changes depending on the initial state. 


\section{CONCLUSION}

We described an alternative approach to quantum Brownian motion, based on the Heisenberg picture. There are three essential ingredients: (i) the full Hamiltonian (1), describing both the evolution of the system and of the bath, (ii) an uncorrelated initial state for the system and the bath (3), and (iii) the spectral density (2), which has to satisfy precise physical constraints. ${ }^{3}$

Starting from these ingredients, we derived explicitly the adjoint master equation (21) for a generic operator of the system. Due to the specific structure of the characteristic operator, from the adjoint master equation we obtained the more familiar master equation for the statistical operator (36). In general, this procedure is not straightforward; however, in this case it was possible to carry out the calculations analytically. As expected, the master equation we obtained is equivalent to previous results $[30,33]$.

A criterion for the complete positivity of the dynamics was given. This becomes important when approximations are needed to carry out calculations and then complete positivity is not guaranteed anymore.

The two approaches (Heisenberg and Schrödinger) are equivalent; however, the explicit expression of the coefficients of the master equation, in the original framework of Eq. (5), can be given only in the weak-coupling regime [33], whereas for the approach here presented it can be given for more general and physically relevant situations [6]. A similar result was obtained in [43]; however, there is an important difference with respect to our approach: Differently from [43], we are not bound to computing the time evolution of the state of the system, which in general is a complicated task. The explicit dependence on the initial state appears only in the initial expectation values and not in the dynamics. This simplifies the derivation of expectation values of physical quantities and even more it makes the latter possible also for nontrivial states such as a Gaussian state.

\section{ACKNOWLEDGMENTS}

The authors acknowledge financial support from the University of Trieste (Grant FRA 2016) and INFN.

\section{APPENDIX A: EXPLICIT FORM OF $\phi(t)$}

With reference to Eq. (17), it is easy to see that for $\hat{\rho}_{\mathrm{B}}$ as in Eq. (18) the trace over $\hat{\chi}_{\beta}(t)$ gives a real and positive function of time. Using the definition of the spectral density in Eq. (2), one immediately derives $\phi(t)=\lambda^{2} \phi_{1}(t)+\mu^{2} \phi_{2}(t)+$ $\lambda \mu \phi_{3}(t)$, where the explicit form of $\phi_{i}(t)$ is

$$
\begin{aligned}
& \phi_{1}(t)=-\int_{0}^{t} d t^{\prime} \int_{0}^{t} d s \frac{D_{1}\left(t^{\prime}-s\right)}{4 M^{2}} G_{2}\left(t-t^{\prime}\right) G_{2}(t-s), \\
& \phi_{2}(t)=-\frac{1}{4} \int_{0}^{t} d t^{\prime} \int_{0}^{t} d s D_{1}\left(t^{\prime}-s\right) G_{1}\left(t-t^{\prime}\right) G_{1}(t-s),
\end{aligned}
$$

\footnotetext{
${ }^{3}$ The most important physical constraint on the spectral density is dictated by the validity of the fluctuation-dissipation theorem (A3) for the dissipative $D(t)$ and noise $D_{1}(t)$ kernels.
}

$$
\phi_{3}(t)=-\frac{G_{2}(t)}{2 M} \int_{0}^{t} d s D_{1}(s) G_{2}(t-s),
$$

with

$$
D_{1}(t)=2 \hbar \int_{0}^{+\infty} d \omega J(\omega) \operatorname{coth}(\beta \hbar \omega / 2) \cos (\omega t)
$$

defining the noise kernel. Here $D_{1}(t)$ is related to the dissipative kernel $D(t)$ through the fluctuation-dissipation theorem [57-61]

$$
\begin{aligned}
& \int_{-\infty}^{+\infty} d t \cos (\omega t) D_{1}(t) \\
& \quad=\operatorname{coth}\left(\frac{\beta \hbar \omega}{2}\right) \int_{-\infty}^{+\infty} d t \sin (\omega t) D(t) .
\end{aligned}
$$

\section{APPENDIX B: EXPLICIT FORM OF THE ADJOINT MASTER EQUATION}

Starting from Eqs. (16) for $\alpha_{1}(t)$ and $\alpha_{2}(t)$, linear combinations of these relations give the following relations:

$$
\begin{aligned}
& \lambda \hat{\chi}_{t}=-\frac{M \dot{G}_{1}(t)}{F(t)}\left[\hat{\chi}_{t}, \hat{x}\right]-\frac{\dot{G}_{2}(t)}{F(t)}\left[\hat{\chi}_{t}, \hat{p}\right], \\
& \mu \hat{\chi}_{t}=\frac{G_{1}(t)}{F(t)}\left[\hat{\chi}_{t}, \hat{x}\right]+\frac{G_{2}(t)}{M F(t)}\left[\hat{\chi}_{t}, \hat{p}\right],
\end{aligned}
$$

where we defined

$$
F(t)=\hbar\left[G_{1}(t) \dot{G}_{2}(t)-\dot{G}_{1}(t) G_{2}(t)\right] .
$$

By combining the results in Eqs. (19) and (B1), one immediately can check that Eq. (19) takes the Lindblad timedependent form described in Eq. (21). In particular, the effective Hamiltonian $\hat{H}_{\text {eff }}$ is given by Eq. (23), where

$$
\begin{aligned}
\Gamma^{\mathrm{A}}(t) & =\frac{\hbar}{2} \frac{G_{1}(t) \ddot{G}_{2}(t)-\ddot{G}_{1}(t) G_{2}(t)}{F(t)}, \\
\Delta^{\mathrm{A}}(t) & =\hbar \frac{\dot{G}_{1}(t) \ddot{G}_{2}(t)-\ddot{G}_{1}(t) \dot{G}_{2}(t)}{F(t)}
\end{aligned}
$$

and the elements of the Kossakowski matrix $K_{a, b}(t)$ are

$$
\begin{aligned}
K_{11}(t)= & \frac{1}{\hbar F(t)} \int_{0}^{t} d s D_{1}(s)\left[G_{1}(t) \dot{G}_{2}(t-s)\right. \\
& \left.-\dot{G}_{1}(t) G_{2}(t-s)\right], \\
K_{12}(t)= & \frac{1}{2 M \hbar F(t)} \int_{0}^{t} d s D_{1}(s)\left[G_{1}(t-s) G_{2}(t)\right. \\
& \left.-G_{1}(t) G_{2}(t-s)\right]-i \frac{\Gamma^{\mathrm{A}}(t)}{\hbar},
\end{aligned}
$$

and $K_{22}(t)=0$.

\section{APPENDIX C: DERIVATION OF THE MASTER EQUATION FOR THE STATES}

To construct $\tilde{\mathbb{L}}_{t}$, we start from the derivative with respect to the parameters $\lambda$ and $\mu$ of the characteristic operator $\hat{\chi}_{t}$ [see Eq. (15)],

$$
\frac{\partial}{\partial \lambda} \hat{\chi}_{t}=i G_{1}(t) \hat{x} \hat{\chi}_{t}+i \frac{G_{2}(t)}{M} \hat{p} \hat{\chi}_{t}+A(t) \hat{\chi}_{t}
$$


and

$$
\frac{\partial}{\partial \mu} \hat{\chi}_{t}=i M \dot{G}_{1}(t) \hat{x} \hat{\chi}_{t}+i \dot{G}_{2} \hat{p} \hat{\chi}_{t}+B(t) \hat{\chi}_{t},
$$

where

$$
\begin{aligned}
& A(t)=\left(\frac{i}{2} F(t)+\phi_{3}(t)\right) \mu+2 \phi_{1}(t) \lambda, \\
& B(t)=\left(-\frac{i}{2} F(t)+\phi_{3}(t)\right) \lambda+2 \phi_{2}(t) \mu,
\end{aligned}
$$

with $F(t)$ defined in Eq. (B2). By linearly combining Eqs. (C1) and $(\mathrm{C} 2)$ we arrive at the following expressions:

$$
\begin{aligned}
\hat{x} \hat{\chi}_{t}= & \frac{i \hbar}{M F(t)} G_{2}(t) \frac{\partial}{\partial \mu} \hat{\chi}_{t}-\frac{i \hbar}{F(t)} \dot{G}_{2}(t) \frac{\partial}{\partial \lambda} \hat{\chi}_{t} \\
& +\frac{i \hbar}{F(t)}\left[\dot{G}_{2}(t) A(t)-\frac{G_{2}(t)}{M} B(t)\right] \hat{\chi}_{t}, \\
\hat{p} \hat{\chi}_{t}= & -\frac{i \hbar}{F(t)} G_{1}(t) \frac{\partial}{\partial \mu} \hat{\chi}_{t}+\frac{i \hbar}{F(t)} M \dot{G}_{1}(t) \frac{\partial}{\partial \lambda} \hat{\chi}_{t} \\
& -\frac{i \hbar}{F(t)}\left[M \dot{G}_{1}(t) A(t)-G_{1}(t) B(t)\right] \hat{\chi}_{t},
\end{aligned}
$$

which we use to replace the terms proportional to $\hat{x}$ and $\hat{p}$ in Eq. (19); the right-hand side gives $\mathbb{L}_{t}\left[\hat{\chi}_{t}\right]=\mathbb{L}_{t} \circ \boldsymbol{\Phi}_{t}[\hat{\chi}(0)]$. By multiplying it from the left with $\boldsymbol{\Phi}_{t}^{-1}$ we obtain

$$
\begin{aligned}
& \boldsymbol{\Phi}_{t}^{-1} \circ \mathbb{L}_{t} \circ \boldsymbol{\Phi}_{t}[\hat{\chi}(0)] \\
& =\tilde{\mathbb{L}}_{t}[\hat{\chi}(0)]=\boldsymbol{\Phi}_{t}^{-1}\left[M \Delta^{\mathrm{A}}(t) \mu\left(A(t)-\frac{\partial}{\partial \lambda}\right)\right. \\
& \quad+\left(\frac{\lambda}{M}+2 \mu \Gamma^{\mathrm{A}}(t)\right)\left(\frac{\partial}{\partial \mu}-B(t)\right) \\
& \left.\quad+\frac{i \hbar}{2}\left[\dot{\alpha}_{1}(t) \alpha_{2}(t)-\alpha_{1}(t) \dot{\alpha}_{2}(t)\right]+\dot{\phi}(t)\right] \boldsymbol{\Phi}_{t}[\hat{\chi}(0)],
\end{aligned}
$$

where $\Delta^{\mathrm{A}}(t)$ and $\Gamma^{\mathrm{A}}(t)$ are defined in Eqs. (B3). Now the expression within the square brackets contains no operator; therefore, the action of the inverse map $\boldsymbol{\Phi}_{t}^{-1}$ and of the direct map $\boldsymbol{\Phi}_{t}$ cancel each other. Moreover, because of Eqs. (C1) and (C2) we have

$$
\begin{aligned}
\frac{\partial}{\partial \lambda} \hat{\chi}(0) & =i\left(\hat{x}+\frac{\hbar}{2} \mu\right) \hat{\chi}(0), \\
\frac{\partial}{\partial \mu} \hat{\chi}(0) & =i\left(\hat{p}-\frac{\hbar}{2} \lambda\right) \hat{\chi}(0)
\end{aligned}
$$

and therefore Eq. (C5) becomes

$$
\begin{aligned}
\tilde{\mathbb{L}}_{t}[\hat{\chi}(0)]= & \left\{M \Delta^{\mathrm{A}}(t) \mu\left[A(t)-i\left(\hat{x}+\frac{\hbar}{2} \mu\right)\right]\right. \\
& +\left(\frac{\lambda}{M}+2 \mu \Gamma^{\mathrm{A}}(t)\right)\left[i\left(\hat{p}-\frac{\hbar}{2} \lambda\right)-B(t)\right] \\
& \left.+\frac{i \hbar}{2}\left[\dot{\alpha}_{1}(t) \alpha_{2}(t)-\alpha_{1}(t) \dot{\alpha}_{2}(t)\right]+\dot{\phi}(t)\right\} \hat{\chi}(0) .
\end{aligned}
$$

Now we want to rewrite the above relation without any explicit dependence on $\lambda$ and $\mu$. In order to do so, we use the same procedure used in passing from Eq. (19) to Eq. (21). According to Eq. (22),

$$
[\hat{\chi}(0), \hat{x}]=\hbar \mu \hat{\chi}(0), \quad[\hat{\chi}(0), \hat{p}]=-\hbar \lambda \hat{\chi}(0),
$$

which, together with Eqs. (C7) and (C6), gives the explicit form of $\tilde{\mathbb{L}}_{t}$ reported in Eq. (33), where

$$
\begin{aligned}
\tilde{K}_{11}(t)= & -\frac{2}{\hbar^{2}}\left[\dot{\phi}_{2}(t)-4 \phi_{2}(t) \Gamma^{\mathrm{A}}(t)+M \Delta^{\mathrm{A}}(t) \phi_{3}(t)\right], \\
\tilde{K}_{22}(t)= & 0 \\
\tilde{K}_{12}(t)= & \frac{1}{\hbar^{2}}\left[\dot{\phi}_{3}(t)-\frac{2}{M} \phi_{2}(t)+2 M \Delta^{\mathrm{A}}(t) \phi_{1}(t)\right. \\
& \left.-2 \phi_{3}(t) \Gamma^{\mathrm{A}}(t)\right]-\frac{i}{\hbar} \Gamma^{\mathrm{A}}(t) .
\end{aligned}
$$

\section{APPENDIX D: EXPLICIT EXPRESSION FOR $\Lambda^{\operatorname{dif}}(t)$ AND $E(t)$}

Following the procedure described in the main text, we can derive the solutions for the quadratic combinations of the position and momentum operators. Starting from Eq. (21), one applies $\mathbb{L}_{t}$ to the unitary evolved operator $\hat{O}(t)$ written in terms of $\hat{x}$ and $\hat{p}$. Then one applies in Eq. (21) the commutation relations between the operators at time $t=0$ and finds $\mathbb{L}_{t}\left[\hat{O}_{t}\right]$, depending only on operators at time $t=0$. For example, in the case of $\hat{x}^{2}$ this reads

$$
\begin{aligned}
\mathbb{L}_{t}\left[\hat{x}_{t}^{2}\right]= & 2 \dot{G}_{1}(t) \dot{G}_{2}(t) \hat{x}^{2}+2 \dot{G}_{1}(t) \dot{G}_{2}(t) \hat{p}^{2} / M^{2} \\
& +\left[G_{1}(t) \dot{G}_{2}(t)+\dot{G}_{1}(t) G_{2}(t)\right]\{\hat{x}, \hat{p}\} / M-2 \dot{\phi}_{1}(t) .
\end{aligned}
$$

Then one integrates the obtained expression and finds the evolution of $\hat{O}_{t}$ under the reduced dynamics. In the case of the quadratic combinations of the position and momentum operators the solutions are

$$
\begin{aligned}
\hat{x}_{t}^{2}= & G_{1}^{2}(t) \hat{x}^{2}+\frac{G_{1}(t) G_{2}(t)}{M}\{\hat{x}, \hat{p}\} \\
& +\frac{1}{M^{2}} G_{2}^{2}(t) \hat{p}^{2}-2 \phi_{1}(t), \\
\left\langle\{\hat{x}, \hat{p}\}_{t}\right\rangle= & 2 M \dot{G}_{1}(t) \dot{G}_{2}(t)\left\langle\hat{x}^{2}\right\rangle+\frac{2}{M} G_{1}(t) G_{2}(t)\left\langle\hat{p}^{2}\right\rangle \\
& +\left[G_{1}(t) \dot{G}_{2}(t)+\dot{G}_{1}(t) G_{2}(t)\right]\langle\{\hat{x}, \hat{p}\}\rangle-2 \phi_{3}(t), \\
\left\langle\hat{p}_{t}^{2}\right\rangle= & M^{2} \dot{G}_{1}^{2}(t)\left\langle\hat{x}^{2}\right\rangle+M \dot{G}_{1}(t) \dot{G}_{2}(t)\langle\{\hat{x}, \hat{p}\}\rangle \\
& +\dot{G}_{2}^{2}(t)\left\langle\hat{p}^{2}\right\rangle-2 \phi_{2}(t) .
\end{aligned}
$$

Then we can compute how the system diffuses in space $\Lambda^{\mathrm{dif}}(t)=\left\langle\hat{x}_{t}^{2}\right\rangle-\left\langle\hat{x}_{t}\right\rangle^{2}$ :

$$
\begin{aligned}
\Lambda^{\operatorname{dif}}(t)= & G_{1}^{2}(t)\left(\left\langle\hat{x}^{2}\right\rangle-\langle\hat{x}\rangle^{2}\right)+\frac{G_{2}^{2}(t)}{M^{2}}\left(\left\langle\hat{p}^{2}\right\rangle-\langle\hat{p}\rangle^{2}\right) \\
& +\frac{2 G_{1}(t) G_{2}(t)}{M}\left(\frac{\langle\{\hat{p}, \hat{x}\}\rangle}{2}-\langle\hat{p}\rangle\langle\hat{x}\rangle\right)-2 \phi_{1}(t) .
\end{aligned}
$$


The energy $E(t)=\left\langle\hat{p}_{t}^{2}\right\rangle / 2 M+\frac{1}{2} M \omega_{\mathrm{S}}^{2}\left\langle\hat{x}_{t}^{2}\right\rangle$ of the system $S$ is

$$
\begin{aligned}
E(t)= & \frac{M}{2}\left[\omega_{\mathrm{S}}^{2} G_{1}^{2}(t)+\dot{G}_{1}^{2}(t)\right]\left\langle\hat{x}^{2}\right\rangle+\frac{\omega_{\mathrm{S}}^{2} G_{2}^{2}(t)+\dot{G}_{2}^{2}(t)}{2 M}\left\langle\hat{p}^{2}\right\rangle+\frac{1}{2}\left[\omega_{\mathrm{S}}^{2} G_{1}(t) G_{2}(t)+\dot{G}_{1}(t) \dot{G}_{2}(t)\right]\langle\{\hat{x}, \hat{p}\}\rangle \\
& -\left(\frac{\phi_{2}(t)}{M}+M \omega_{\mathrm{S}}^{2} \phi_{1}(t)\right) .
\end{aligned}
$$

[1] U. Weiss, Quantum Dissipative Systems, 2nd ed. (World Scientific, Singapore, 1999).

[2] H. P. Breuer and F. Petruccione, The Theory of Open Quantum Systems (Oxford University Press, Oxford, 2002).

[3] E. Joos et al., Decoherence and the Appearance of a Classical World in Quantum Theory, 2nd ed. (Springer, Berlin, 2003).

[4] C. Gardiner and P. Zoller, Quantum Noise (Springer, Berlin, 2004).

[5] M. A. Schlosshauer, Decoherence and the Quantum-ToClassical Transition, 1st ed. (Springer, Berlin, 2007).

[6] L. Diósi and L. Ferialdi, Phys. Rev. Lett. 113, 200403 (2014).

[7] A. Pomyalov and D. J. Tannor, J. Chem. Phys. 123, 204111 (2005).

[8] J. J. Hope, Phys. Rev. A 55, R2531(R) (1997).

[9] H. P. Breuer, D. Faller, B. Kappler, and F. Petruccione, Europhys. Lett. 54, 14 (2001).

[10] X. Q. Jiang, B. Zhang, Z. W. Lu, and X. D. Sun, Phys. Rev. A 83, 053823 (2011).

[11] G. D. Scholes, G. R. Fleming, A. Olaya-Castro, and R. van Grondelle, Nat. Chem. 3, 763 (2011).

[12] R. E. Blankenship et al., Science 332, 805 (2011).

[13] F. Fassioli, A. Olaya-Castro, and G. D. Scholes, J. Phys. Chem. Lett. 3, 3136 (2012).

[14] N. Lambert et al., Nat. Phys. 9, 10 (2013).

[15] J. Reichel, W. Hänsel, P. Hommelhoff, and T. W. Hänsch, Appl. Phys. B 72, 81 (2001).

[16] M. Paternostro et al., New J. Phys. 8, 107 (2006).

[17] D. Hunger, S. Camerer, T. W. Hänsch, D. König, J. P. Kotthaus, J. Reichel, and P. Treutlein, Phys. Rev. Lett. 104, 143002 (2010).

[18] S. Gröblacher, A. Trubarov, N. Prigge, G. D. Cole, M. Aspelmeyer, and J. Eisert, Nat. Commun. 6, 7606 (2015).

[19] M. Toda, J. Phys. Soc. Jpn. 13, 1266 (1958).

[20] V. Magalinskii, Zh. Eksp. Tero. Fiz. 36, 1382 (1959).

[21] I. R. Senitzky, Phys. Rev. 119, 670 (1960).

[22] J. Schwinger, J. Math. Phys. 2, 407 (1961).

[23] R. P. Feynman and F. L. J. Vernon, Ann. Phys. (N.Y.) 24, 118 (1963).

[24] G. Ford, M. Kac, and P. Mazur, J. Math. Phys. 6, 504 (1965).

[25] P. Ullersma, Physica 32, 27 (1966).

[26] A. O. Caldeira and A. J. Leggett, Physica A 121, 587 (1983).

[27] H. Grabert, U. Weiss, and P. Talkner, Z. Phys. B 55, 87 (1984).

[28] K. Lindenberg and B. J. West, Phys. Rev. A 30, 568 (1984).
[29] P. S. Riseborough, P. Hanggi, and U. Weiss, Phys. Rev. A 31, 471 (1985).

[30] F. Haake and R. Reibold, Phys. Rev. A 32, 2462 (1985).

[31] G. W. Ford and M. Kac, J. Stat. Phys. 46, 803 (1987).

[32] W. G. Unruh and W. H. Zurek, Phys. Rev. D 40, 1071 (1989).

[33] B. L. Hu, J. P. Paz, and Y. Zhang, Phys. Rev. D 45, 2843 (1992).

[34] L. Ferialdi, Phys. Rev. A 95, 020101 (2017).

[35] A. Kossakowski, Rep. Math. Phys. 3, 247 (1972).

[36] V. Gorini, A. Kossakowski, and E. C. G. Sudarshan, J. Math. Phys. 17, 821 (1976).

[37] G. Lindblad, Commun. Math. Phys. 48, 119 (1976).

[38] V. Ambegaokar, Ber. Bunsenges. Phys. Chem. 95, 400 (1991).

[39] L. Diósi, Europhys. Lett. 22, 1 (1993).

[40] F. Haake and M. Lewenstein, Phys. Rev. A 28, 3606 (1983).

[41] U. Geigenmüller, U. Titulaer, and B. Felderhof, Physica A 119, 41 (1983).

[42] M. Frimmer, J. Gieseler, and L. Novotny, Phys. Rev. Lett. 117, 163601 (2016).

[43] G. W. Ford and R. F. O'Connell, Phys. Rev. D 64, 105020 (2001).

[44] S. Ghose and B. C. Sanders, J. Mod. Opt. 54, 855 (2007).

[45] E. S. Gómez, G. Cañas, E. Acuña, W. A. T. Nogueira, and G. Lima, Phys. Rev. A 91, 013801 (2015).

[46] K.-j. Huang, Y. Yan, J.-p. Zhu, Y.-f. Xiao, and G.-x. Li, Phys. Rev. A 93, 033832 (2016).

[47] J. von Neumann, Math. Ann. 104, 570 (1931).

[48] B. J. Hiley, J. Comput. Electron. 14, 869 (2015).

[49] L. Ferialdi, Phys. Rev. Lett. 116, 120402 (2016).

[50] F. Benatti, Dynamics, Information and Complexity in Quantum Systems (Springer Netherlands, Dordrecht, 2009).

[51] B. Demoen, P. Vanheuverzwijn, and A. Verbeure, Lett. Math. Phys. 2, 161 (1977).

[52] T. Heinosaari, A. S. Holevo, and M. M. Wolf, Quantum Inf. Comput. 10, 0619 (2010).

[53] J. J. Halliwell and T. Yu, Phys. Rev. D 53, 2012 (1996).

[54] J. J. Halliwell, J. Phys. A 40, 3067 (2007).

[55] M. Wendling et al., J. Phys. Chem. B 104, 5825 (2000).

[56] R. P. Feynman and A. R. Hibbs, Quantum Physics and Path Integrals (McGraw-Hill, New York, 1965).

[57] H. B. Callen and T. A. Welton, Phys. Rev. 83, 34 (1951).

[58] H. B. Callen and R. F. Greene, Phys. Rev. 86, 702 (1952).

[59] R. Kubo, Rep. Prog. Phys. 29, 255 (1966).

[60] N. Pottier and A. Mauger, Physica A 291, 327 (2001).

[61] Y. Yan and R. Xu, Annu. Rev. Phys. Chem. 56, 187 (2005). 